\title{
Théologiques
}

Théologiques

\section{Le sens de la vie}

\section{Une question assez récente, mais pleine de saveur}

\section{Jean Grondin}

Volume 9, numéro 2, automne 2001

Sens et spiritualité dans les pratiques professionnelles

URI : https://id.erudit.org/iderudit/007292ar

DOI : https://doi.org/10.7202/007292ar

Aller au sommaire du numéro

Éditeur(s)

Faculté de théologie de l'Université de Montréal

ISSN

1188-7109 (imprimé)

1492-1413 (numérique)

Découvrir la revue

Citer cet article

Grondin, J. (2001). Le sens de la vie : une question assez récente, mais pleine de saveur. Théologiques, 9(2), 7-15. https://doi.org/10.7202/007292ar d'utilisation que vous pouvez consulter en ligne.

https://apropos.erudit.org/fr/usagers/politique-dutilisation/ 


\title{
Le sens de la vie.
}

\section{Une question assez récente, mais pleine de saveur}

\author{
Jean GRONDIN \\ Département de philosophie \\ Université de Montréal
}

Albert Camus écrit au début du Mythe de Sisyphe qu'il n'y a qu'un problème philosophique vraiment sérieux, celui de savoir si la vie mérite d'être vécue. Se demander si la vie mérite d'être vécue revient, aujourd'hui, à se demander si la vie peut avoir un sens. À cette question, j'aimerais d'abord répondre, de manière assez péremptoire, que, pour une raison bien simple, la vie ne peut pas ne pas avoir de sens. En effet, de deux choses l'une : ou bien la vie a un (ou des) sens, ou bien elle n'en a pas. Mais si elle n'en a pas, si la vie est " absurde ", comme l'a pensé la génération de Camus, c'est parce que l'on présuppose qu'elle doit avoir un sens. En effet, la vie ne peut être ressentie, et très justement souvent, comme "insensée " qu'à l'aune d'une attente de sens. C'est parce que la vie devrait en avoir un que l'on peut parler d'une vie qui n'a pas de sens. En un sens, les penseurs de l'absurde sont donc les philosophes les plus « rationalistes » qui soient. C'est parce qu'ils prêtent un sens très fort à la vie qu'ils proclament l'absurdité de l'existence. C'est pourquoi personne ne croit peut-être davantage au sens de l'existence que ceux qui le contestent.

La même chose est vraie, du reste, du pessimiste, qui se raconte que tout va mal tourner. Mais s'il le pense et le dit (avant un examen difficile, par exemple), c'est parce qu'il espère secrètement que les choses vont finir par bien aller, comme si le pessimiste espérait, et de fait il le fait, se tromper en s'attendant au pire. C'est pourquoi le philosophe Hans-Georg Gadamer a toujours dit que le pessimiste manquait un peu de probité ${ }^{1}$ : il se ment et cherche à se mentir à lui-même en attendant le pire, mais dans l'espoir inavoué du meilleur.

1. Voir par exemple l'interview « Die Kindheit wacht auf. Gespräch mit dem Philosophen Hans-Georg Gadamer », dans Die Zeit, 13 ( 26 mars 1993), p. 23. 
Il en va un peu de cette manière avec la question du sens de la vie. La question repose elle-même sur une attente de sens, en sorte que la vie, dès lors qu'elle s'interroge sur son sens, ne peut pas ne pas le présupposer. Reste à savoir en quoi il consiste.

Avant de tenter de répondre à cette question, il est peut-être important de rappeler que la question du sens de la vie ne s'est pas toujours posée de manière aussi dramatique qu'aujourd'hui. Il s'agit, en effet, d'une question beaucoup plus récente qu'on ne le pense d'ordinaire. Un chercheur allemand ${ }^{2}$ a d'ailleurs rappelé que le premier à avoir employé la formule aurait été nul autre que Friedrich Nietzsche (1844-1900). Si cette situation est assez ironique, c'est que Nietzsche, le grand penseur de la "mort de Dieu », est généralement perçu comme celui qui aurait vigoureusement contesté que la vie ait un sens! En fait, il semble avoir été le tout premier à parler expressément d'un sens de la vie.

Nietzsche l'a fait dans un texte de 1875 , donc un texte de jeunesse, qu'il n'a pas lui-même publié et qui se retrouve dans l'édition de ses œuvres posthumes. Et dans le texte de Nietzsche, la formule du « sens de la vie », comme cela est toujours vrai des premières occurrences, n'est pas particulièrement appuyée ou mise en évidence, en sorte que son sens précis reste assez difficile à cerner. Voici le texte en question :

La plupart des hommes ne se considèrent même pas comme des individus ; c'est ce que montre leur vie (...) L'homme n'est un individu que selon trois formes d'existence : comme philosophe, comme saint ou comme artiste. Il n'est que de voir avec quoi un homme de science tue sa propre vie : qu'estce que la doctrine des particules chez les Grecs peut bien avoir à voir avec le sens de la vie ? On voit ici jusqu'à quel point d'innombrables hommes ne vivent que pour préparer un homme véritable : les philologues, par exemple, ne sont là que pour préparer le philosophe, lequel sait profiter de leur travail de fourmi pour dire quelque chose à propos de la valeur de la vie. Il va, bien sûr, de soi que, sans cette direction, la plus grande partie de ce travail de fourmi est absolument insensée et superflue ${ }^{3}$.

On ne proposera pas ici une exégèse détaillée du texte de Nietzsche. Il est clair, en tout cas, que dans ce texte, le jeune Nietzsche exalte trois

2. Il s'agit de V. GERHARDT, dans son livre Friedrich Nietzsche, Munich, Beck, 1992, p. 21. Il a été suivi par G. FigAL, Nietzsche. Eine philosophische Einführung, Stuttgart, Reclam, 1999, p. 135.

3. F. NIETZSCHE, Sämtliche Werke. Kritische Studienausgabe / édité par G COlli et M. MontinARI, München/Berlin/New York, De Gruyter, 1986, t. 8 . 
formes de vie ou d'existence, celles du philosophe, de l'artiste et du saint (ce qui peut aussi surprendre sous sa plume, mais les grands philosophes ne se conforment jamais aux images très simplistes que l'on se fait à leur propos !). On devine aisément pourquoi : dans les trois cas, la forme d'existence a un sens qui colle de très près à la vie de l'individu : le philosophe, l'artiste et le saint sont un peu les « artisans » de leur destin et le sont, du moins aime-t-on le supposer, dans l'ensemble de leur existence. C'est pourquoi la vie des saints, des artistes et des vrais philosophes peut très souvent être un modèle aussi, sinon plus inspirant encore que leurs œuvres mêmes (ce qui est certainement le cas de Socrate ou de Jésus, qui n'ont rien écrit). On s'intéresse en tout cas davantage à la vie de Rembrandt, de Mozart ou de saint François d'Assise qu'à celle des grands scientifiques. Nietzsche semble donc associer cette notion d'un sens de la vie à une conception forte de l'individualité, qui caractérise aussi assez généralement sa pensée, mais aussi notre époque en général : la vie n'a de sens que pour un être qui prend sa propre en vie en main, qui en fait en quelque sorte une œuvre d'art. Tous les autres, dit-on parfois, se " laissent » vivre, ou paraissent s'abandonner à des occupations vaines et futiles (même s'il serait hardi de le prétendre pour autrui, on ne peut toujours parler que pour soi).

Dans un geste qui est sans doute dirigé contre sa propre formation, Nietzsche exclut volontiers les " philologues » de ces individualités pour lesquelles la vie comporte et compose un sens. Les philologues, ce sont les savants qui s'intéressent à l'édition et au commentaire érudit des textes de l'Antiquité classique. Nietzsche était lui-même philologue de formation et enseignait encore à cette époque la philologie classique à l'université de Bâle. Or, les philologues, écrit-il (pour lui-même, manifestement), ne sont que des fourmis qui accomplissent un travail qui ne peut servir qu'aux grandes individualités, celles du philosophe, du saint et de l'artiste, où Nietzsche a certainement voulu célébrer ses propres idéaux de vie.

La signification précise du texte de Nietzsche, son sens des grandes individualités et son aristocratique mépris de l'existence des

Nachgelassene Fragmente 1875-1879, p. 32, N 1875, 3 [63]: « Nur bei drei Existenzformen bleibt der Mensch Individuum : als Philosoph, Heiliger und Künstler. Man sehe nur, womit ein wissenschaftlicher Mensch sein Leben todt schlägt : was hat die griechische Partikellehre mit dem Sinne des Lebens zu tun ?». Je souligne. 
"fourmis ", nous intéresse moins ici que le sens de la formule qu'il semble avoir été le premier à risquer, celle du " sens de la vie ». Nietzsche a beau s'en prendre aux philologues, il n'est sans doute pas indifférent que la formule ait d'abord été utilisée par un philologue de profession. C'est que la formule, inouie, d'un sens de la vie présuppose que la vie peut être "lue » comme un " texte ». Tout comme un texte écrit, ou dit, la vie possède un commencement, une fin et par là même une direction et un sens. Elle peut dès lors être considérée comme un "parcours sensé "(tel un cursus, d'où l'idée de " carrière "), susceptible de direction et de souci, mais aussi de renversements et de catastrophes. La question du sens de la vie est celle de savoir si cette trame ou cette extension ont un sens et si oui, lequel.

On pourrait parler ici d'une «philologisation » de l'existence. La vie humaine apparaît, en effet, comme un « texte » susceptible de bénéficier d'un sens. Ce sens est-il immanent à la vie, doit-il lui être insufflé, faut-il lui en inventer, lui en prescrire un ? Autant de questions essentielles, dont il faudrait débattre lentement, mais il n'est pas indifférent d'observer que la question du sens de la vie ne se soit posée qu'aussi tardivement. En fait, si la question du sens de la vie se pose aujourd'hui, en tout cas depuis Nietzsche, avec autant d'acuité, c'est parce qu'en un sens, elle a cessé d'en avoir un. Si autrefois la question du sens de la vie ne se posait guère, c'est que ce sens allait un peu de soi. La vie se trouvait et se savait instinctivement enchâssée dans un ordre du monde ou du cosmos, auquel elle n'avait qu'à se conformer ${ }^{4}$, en se pliant à ses rites, qui étaient tous des rites de passage, plus ou moins convenus.

La question du sens de la vie présuppose que ce sens ne va pas, ne va plus de soi. Si cette situation est assez aporétique, c'est parce qu'il paraît bien difficile de donner un sens à la vie lorsque ce sens est devenu aussi problématique. C'est un peu comme lorsque l'on s'interroge sur le sens d'une institution périmée ou d'une relation, amoureuse par exemple. C'est parce qu'elle fait problème et que toutes les tentatives pour lui donner ou lui redonner un sens ne font que l'aggraver. La question du sens de la vie ne peut donc être abordée dans l'insouciance, dont elle est en quelque sorte la tragique nostalgie.

4. Sur cette évidence du sens du monde, voir le livre de R. BRAGUE, La Sagesse du monde. Histoire de l'expérience humaine de l'univers, Paris, Fayard, 1999. 
Si la question du sens de la vie est tragique, c'est que la question est beaucoup plus évidente que la réponse. En un sens, brutal, la question paraît saper toute possibilité de réponse. C'est que toute réponse peut être vue, et déconstruite, comme une réponse construite, donc factice, c'est-à-dire désespérée, à un problème auquel il ne peut y avoir de réponse. C'est ainsi par exemple que les réponses disons " religieuses » (la vie n'a de sens qu'en vue d'un au-delà), " humanistes " (œuvrons à l'avancement de la culture et au progrès de l'humanité) ou vaguement hédonistes (jouissons de la vie, il n'y a qu'elle) à la question du sens de la vie seront perçues comme des tentatives d'apaisement de l'angoisse suscitée par l'horreur de l'existence ou de la mort. Il revient à chacun, soupirait Max Weber, de trouver les démons qui tiendront les fils de son existence. Mais tous les démons sont-ils pareils ? Est-il indifférent de se vouer à Jésus, Bouddha, Karl Marx ou Madonna?

Une chose est sûre, pour la philosophie en tout cas : seule la voie de Socrate, celle de la connaissance de soi ou du dialogue intérieur, est ouverte. C'est parce que la vie est interrogation sur elle-même que chacun doit répondre, au moins une fois dans sa vie, la seule qui nous soit impartie, sans possibilité d'appel, à la question du sens de l'existence dans le temps. Comme il s'agit d'une réponse que je dois me donner à moi-même, à la question que je suis pour moi-même (Augustin), il ne saurait être indifférent de se vouer à un saint plutôt qu'à un autre. C'est qu'il doit s'agir d'un sens dont je puisse répondre, d'autant que ce sens est lui-même réponse à une question, celle que je suis pour moi-même.

De quoi s'enquiert-on lorsque l'on s'interroge sur le sens de la vie ? On a vu que la formule avait d'abord été forgée par un philologue, donc d'un savant dont la profession consiste à s'interroger sur la signification des textes. En quel sens peut-on parler du sens de la vie? Quel est le sens du sens? Dans une visée moins déconstructrice que constructrice, puisqu'ils s'imbriquent l'un dans l'autre, je pense que l'on peut distinguer plusieurs sens du sens dans l'expression, et la recherche, du «sens de la vie »:

1. Le sens possède d'abord, en français comme en plusieurs autres langues, un sens directionnel $:$ il désigne alors simplement la direction d'un mouvement. C'est ainsi que l'on parle du sens des aiguilles d'une montre, du sens du courant ou d'un "sens unique ». 
Appliqué au cas du sens de la vie, on peut dire, provisoirement, que le sens de la vie est celui d'une extension, d'un cursus qui s'étend de la naissance à la mort. Avant de naître, je n'étais pas, « je » n'allais nulle part et ma vie, ou non-vie, n'avait évidemment aucun sens, sinon, à l'extrême limite, pour mes parents qui voulaient un enfant. La vie n'a de sens que parce que je suis né, donc parce que ma naissance est " derrière " moi et que ma vie « va " ou " s'en va » quelque part. Le terme de ce parcours, c'est, bien sûr, la mort, qui est devant moi, qui m'attend, dans toute son implacabilité. Au sens directionnel du terme, le sens de la vie est donc celui d'une course vers la mort, comme l'a martelé Heidegger, une course que nous ne gagnerons évidemment jamais. La formule est paradoxale, et c'est ce paradoxe que nous avons à vivre, mais le sens de la vie, au sens le plus dérisoirement directionnel du terme, c'est la mort. Toute interrogation sur le sens de la vie présuppose cet horizon, terminal, de la mort.

Or le grand paradoxe de la mort, son caractère littéralement insoutenable, est qu'elle signifie — je dis la pire des banalités — la fin de mon existence. La «substance » que je suis, au sens où je suis le substrat de tout ce qui m'arrive, ne sera même plus là pour la subir, la recevoir, l'accueillir. Clic : les lumières s'éteindront, sans moi. Et cette " fin » n'est pas une fin comme les autres, comme lorsque l'on parle de la fin d'un film, d'un repas ou d'un long voyage, car après ces fins, la vie continue. Mais avec la mort, nous ne serons même plus là pour voir comment la vie continue. Nous aurons été et ne "serons " plus rien, en un futur qui résiste même à l'énonciation. Que faire ? En fait, et c'est ce qui est tragique, on ne peut rien faire, car, quoi que l'on fasse, la mort fauchera. Elle nous privera de l'être que nous sommes, mais cette formule est déjà impropre, puisque nous ne serons même plus là pour être privés de quoi que ce soit. Mais c'est de ce "terme " qu'il faut partir si l'on veut s'interroger sur le sens de la vie. La question ne se pose que parce que le sens de la vie, son terme, c'est la mort, qu'on le veuille ou non, et rien ne sert ici de vouloir ou de ne pas vouloir.

2. En plus de ce sens directionnel, qui surplombe toute philosophie du sens de la vie, le sens possède aussi un sens que l'on peut dire " signifiant » ou "significatif », au risque de la tautologie. On parle, en effet, aussi, voire surtout de " sens " pour désigner la signification, l'acception ou la portée d'un mot. Quand un mot ou un discours m'est étranger, je peux, par exemple, consulter un dictionnaire. Le mot qui 
m'apparaissait étranger me devient alors plus familier et d'autant plus familier que je puis l'employer moi-même avec une certaine aisance, comme s'il allait de soi. L'interrogation sur le sens (d'un mot, d'un texte) en est donc une qui peut cesser de se poser quand une nouvelle familiarité se sera installée.

L'interrogation sur le sens de la vie présuppose très certainement aussi un sentiment d'étrangeté, curieux, puisque c'est alors la vie que je vis, que je suis, qui me tient - qui est alors comme étrangère à elle-même. Cette vie que je suis et qui ne cessera qu'avec ma mort a pour moi, malgré sa constante intimité, quelque chose d'étranger, de mystérieux, de déroutant, comme si, sans le savoir, nous étions attachés sur le dos d'un tigre, comme le dit Nietzsche dans un texte foudroyant, que reprendra Foucault dans Les Mots et les Choses. Il est à peu près impossible d'avoir une prise réelle sur soi-même, sur cette extension de la naissance à la mort que nous sommes. Une " prise » n'est possible que vis-à-vis d'un objet qui se trouve en face de nous, ce qui n'est jamais le cas de notre existence. Nous y baignons, le temps d'un soupir. Nul n'est responsable de sa naissance, et la mort reste dans la plupart des cas imprévisible, soudaine et bête. Elle nous rappelle justement que nous sommes des bêtes et que nous périrons comme les fourmis que nous écrasons ou les animaux que nous dévorons. Le défi, que nous sommes, est celui du sens que nous pouvons reconnaître ou donner à notre modeste extension dans le temps. Reconnaître ou donner, on reviendra ailleurs sur cette dualité. Il importe seulement pour l'instant de retenir cette idée, toute simple, que le sens de la vie est celui d'une existence qui soit dotée d'une quelconque "signification ", malgré le non-sens de son terme.

Mais le sens de la vie comporte d'autres sens philosophiquement éclairants et qui pourront nous aider à répondre à la question du sens de la vie face au non-sens. C'est que la notion de sens renvoie non seulement à une direction (1) et une possibilité de signification (2), elle fait aussi appel à une capacité de " sensation ", à un certain "sens » de, mieux, " pour » la vie.

3. C'est que le sens de la vie, c'est aussi, ce que l'on peut appeler, tautologiquement encore une fois, un certain sens " sensitif ", un odorat, un « nez " pour la vie. Le sens désigne ici une capacité de sentir et même de jouir de la vie. Chacun sait que certains en sont ou en paraissent plus capables que d'autres. Intuitivement, on pense volontiers 
que les latins y sont plus aptes que les nordiques, pétris de puritanisme. "Savoir prendre le temps de vivre ", comme on dit, c'est disposer d'un certain "sens " de la vie, savoir reconnaître une certaine saveur à la vie, savoir qui est moins une «connaissance » qu'une capacité ou un être, parfois aussi un bonheur.

Même si Nietzsche a probablement été le premier à parler d'un sens de la vie, cette idée d'une "saveur de la vie ", elle, est très ancienne. On la retrouve, par exemple, chez saint Augustin. Il écrit quelque part que "l'âme a beau exister toujours, elle vit 'plus' si elle est 'sensée' et moins quand elle est 'insensée' » (et quia semper anima est, semper vivit; sed quia magis vivit cum sapit, minusque cum desi$p i t)^{5}$. C'est la traduction française qui parle de «vie sensée » ou non, alors qu'Augustin emploie ici simplement le verbe sapere. C'est un très beau verbe. Dans son premier sens, intransitif, celui auquel on a affaire ici, le verbe sapere veut, en effet, simplement dire qu'une chose " a du goût" (cum sapit). Augustin veut seulement dire que l'âme vit manifestement «plus » si elle a de la saveur que si elle n'en a pas (cum desipit)! Sans trop jouer sur les mots, il va de soi que ce sens intransitif de sapio habite encore le sens transitif du verbe sapere, quand il veut dire "sentir " et même "savoir » quelque chose : je "sais" quelque chose quand j'y trouve quelque saveur. Le contraste établi par Augustin entre sapio (" avoir de la saveur ») et desipio est en tout cas particulièrement lumineux pour illustrer ce sens de la vie : la vie peut avoir du piquant ou être amère, et dès lors être sensée (sapere) ou insensée (desipere).

C'est en ce sens "sensitif » que nous parlons aussi des cinq sens qui nous ouvrent à autrui et au monde. Certains sens sont plus développés que d'autres, certains êtres sont plus sensibles aux odeurs, aux goûts ou aux sons. Mais on parle aussi, en un sens voisin, d'un sens des bonnes manières, d'un sens du tact, d'un sens pour ceci ou pour cela. Dans toutes ces acceptions, le sens désigne une faculté de sentir, un certain "sens de la vie ». La question du sens de la vie, c'est donc aussi cela, la capacité de sentir la vie, de trouver une certaine saveur à la vie et de s'y retrouver dans l'existence. On aurait tort de croire que la philosophie est étrangère à cette sensibilité. En fait, sa fonction

5. Augustin, La Trinité, V, V, 6, Euvres de saint Augustin, t. 15 (Bibliothèque Augustinienne), Paris, Desclée de Brouwer, 1955, p. 432. 
la plus essentielle - même si elle ne s'exerce que très peu aujourd'hui (ce qui n'est un argument que contre l'aujourd'hui) — est peut-être de nous rappeler ce qui rend la vie digne d'être vécue.

Cette idée d'un art de la vie ou d'une sensibilité à la vie, nous amène à évoquer, rapidement, un dernier niveau de sens à cette expression d'un sens de la vie :

4. C'est que l'on entend aussi par "sens ", en un sens un peu plus réflexif, une capacité de juger, une manière de jauger, d'apprécier la vie. C'est ainsi que l'on emploie en français l'expression «à mon sens » pour connoter une certaine appréciation réfléchie des choses. On parlera de même d'un homme de bon sens ou d'un jugement sensé. Le sens se trouve ici accouplé à une certaine sagesse, où se conjuguent l'expérience, la raison et même une certaine simplicité naturelle. La question du sens de la vie aspire à une telle sagesse de vie, qui est la raison d'être, c'est-à-dire l'espoir de toute philosophie. J'essayerai de montrer ailleurs en quoi ce sens de la vie dépend d'un certain sens du Bien ${ }^{6}$.

\begin{abstract}
RÉSUMÉ
Contrairement à ce que l'on pourrait croire, la question du « sens de la vie » est, en fait, assez récente. Elle ne se pose que depuis la fin du XIX ${ }^{\mathrm{e}}$ siècle. Quel est donc le sens de cette question ? Le présent article se propose de faire ressortir les différentes dimensions du « sens » qui est recherché dans cette quête : une direction, mais aussi un foyer, une signification, une capacité de sensation et de réflexion. Ensemble, ces dimensions nous permettent peutêtre de mieux apprécier le sens de la question, et celui de la vie en général.
\end{abstract}

\begin{abstract}
Contrary to popular belief, the question concerning the "meaning of life " is a rather recent one. Actually, it only emerged at the end of the 19th century. What is the meaning of this question? This article attempts to clarify the question by sorting out the different dimensions of the "meaning " that is sought after in this quest : a direction, and thus a focus, a significance, a capacity of sensing and of reflection. Together, they can help us get a better grasp of the meaning of the question, and of life, for that matter.
\end{abstract}

6. JEAN GRONDIN fait paraître en 2003 un livre sur cette question, chez Fides (Montréal). 